\title{
edmetic
}

Revista de Educación Mediática y TIC

Las TIC en la educación, su pertinencia social y educativa

Fecha de recepción: 10/122014

Fecha de revisión: 21/05/2015

Fecha de aceptación: 04/10/2015

edmetic, 5(1), 2016, E-ISSN: 2254-0059; pp.xx-xx

(C) edmetic, Revista de Educación Mediática y TIC 


\section{Las TIC en la educación, su pertinencia social y educativa}

Las Tecnologías de la Información y la Comunicación (TIC) han generado nuevas perspectivas con respecto a los roles del profesor y de los estudiantes, en la redefinición de las metodologías didácticas y de participación, así como de los sistemas de evaluación de los aprendizajes, de las actitudes, de modelos de tutorización, de la pertinencia de la educación en línea, de los procesos de innovación educativa con base en el uso de TIC, entre otros.

En este sentido, mientras algunos se cuestionan sobre el impacto que las TIC han tenido en ciertas figuras sociales, y como deben atender la inserción de estas en su campo de especialidad, otros se cuestionan las características didácticas y curriculares, que deben poseer ciertas aplicaciones 0 metodologías con apoyo en TIC para lograr aprendizajes significativos en sus estudiantes.

Y en línea con lo anterior, hay quiénes analizan la viabilidad de estrategias adecuadas para la integración de las TIC en la práctica educativa, proponiendo para ello, diversas técnicas que puedan apoyar la toma de decisiones en cuanto a los modelos de comportamiento y de metodologías activas que propicien espacios virtuales acordes a las necesidades educativas de los estudiantes. Así como tomar en cuenta la actitud y la percepción que los usuarios tienen con respecto al uso de las TIC y los entornos virtuales.

A partir de estas inquietudes, nos propusimos este monográfico. Mostrar los diversos análisis, prácticas y reflexiones en torno al uso adecuado de las TIC desde una perspectiva de pertinencia social y académica.

El monográfico abre su propuesta con Begoña Sampedro y su análisis teórico de la educación social en el siglo XXI. Resalta la importancia que tiene el educador social en las nuevas realidades sociales en la actualidad, a partir de la inmediatez que poseen las $\mathrm{TIC}$, en especial las redes sociales, que 
influyen de manera significativa en los procesos de socialización, participación ciudadana, desenvolvimiento de la sociedad, así como en el desarrollo comunitario y social. La autora centra su análisis en la titulación universitaria de la misma, en las características básicas de su ocupación profesional, y reflexiona de como las TIC promueven nuevas formas de generación del conocimiento, así como en la conformación de valores y actitudes.

Desde Venezuela, Guillermo Antonio Arraiz, nos presenta como el aprendizaje matemático virtual se ve afectado por diversos factores, a partir de la perspectiva de los estudiantes. En su estudio nos expone la experiencia sobre el desarrollo de un curso introductorio para alumnos de nuevo ingreso en su modalidad mixta a través de la plataforma Moodle, relacionada con el desarrollo del pensamiento lógico matemático. El autor nos señala que los factores afectivos y emocionales, estrategias metacognitivas y cognitivas del aprendizaje, de interacción y comunicación, de la interacción del participante y el entorno virtual, de la acción tutorial del facilitador de matemáticas, son elementos medulares para la reconstrucción teórica del aprendizaje matemático en la virtualidad.

Fredy Forero et al, nos presentan la experiencia del uso de las TIC en tres escuelas multigrado de un área rural en Colombia. Las características particulares de estos cursos multigrado, propicia la necesidad de reflexionar sobre las estrategias más coherentes y pertinentes para la integración las TIC en la práctica educativa, con el fin de lograr una formación integral de los estudiantes. Ya que la sola presencia de los computadores, no garantiza en sí, aprendizajes significativos en los sujetos. En este caso, el análisis del discurso informático, del tipo de interacción con la computadora, de los recursos informáticos utilizados, y de la innovación, ofrece una panorámica de la percepción que los docentes poseen con respecto a las TIC y su práctica educativa.

Las técnicas de minería de datos nos permiten identificar rutas de aprendizaje de los estudiantes, en cursos en línea a través de plataformas 
virtuales de aprendizaje, como Moodle. La exposición del comportamiento que ha tenido el estudiante en la plataforma, nos muestra su proceso de aprendizaje, lo cual permite adaptar el curso a la forma en cómo trabajan los estudiantes, y así tomar medidas ante los problemas que se puedan detectar. Esta situación apoya la toma de consciencia con respecto a lo que sucede en la educación a distancia, a fin de motivar o recomendar rutas de aprendizaje a los estudiantes. Incidiendo en la mejora de calidad y la rentabilidad en el proceso educativo, como nos explican en su propuesta Alejandro Bogarín et al.

El trabajo de Francisco Morales, nos invita a reflexionar sobre el uso de metodologías activas para la adquisición de competencias transversales requeridas dentro del Espacio Europeo de Educación Superior. El estudio se enmarca en el proyecto de "Educación transversal para la solidaridad en la formación de psicólogos y educadores", en donde la meta es fomentar la solidaridad, el voluntariado y la concienciación en universitarios. El autor nos muestra que es necesaria la atención en el diseño y desarrollo adecuado de estrategias activas, así como de una evaluación a través de rúbricas integradoras, que demuestren la eficacia e incidencia de estas, no tan solo en el rendimiento académico sino también de procedimientos, valores y actitudes de los estudiantes.

La utilización de las TIC en el marco del Espacio Europeo de Educación Superior, ha generado compromisos y exigencias encaminadas a mejorar la calidad tanto de los sistemas educativos como de la educación. A partir de esta prerrogativa, Marina Morales et al, nos muestran la percepción que profesores y estudiantes universitarios tienen con respecto a las posibilidades que ofrecen las TIC y los usos que hacen de estas en el proceso enseñanza aprendizaje. Los autores enmarcan su estudio a partir de la reflexión en torno a la formación y alfabetización del profesorado, la actitud del docente hacia las TIC, la alfabetización digital y la tecnofobia docente. 
En línea con el estudio anterior, Carlos de Castro et al, nos comparten la necesidad de que el uso de las TIC se realicen desde una integración curricular al proceso educativo. Tal es el caso del método Smartick, y la forma en cómo se ha integrado en los entornos educativos a nivel de primaria, a fin de potencializar el desarrollo de la competencia matemática. Nos muestran que es necesario que en el aprendizaje de las matemáticas, se aspire al desarrollo de todas las capacidades matemáticas fundamentales, que en algunos casos supondrá realizar actividades para el desarrollo de destrezas básicas, y en otros, al desarrollo de capacidades de razonamiento o de matematización, que irían perfiladas a una línea más constructivista.

Fernanda Araujo et al, traen a la palestra una investigación relacionada con las modalidades - presencial y en línea - en la educación superior y las posibilidades de convergencia entre ellas en Brasil. Las autoras nos muestran un análisis teórico de las políticas públicas, en atención especial al decreto 4.059/2004. Reflexionan desde la teoría, la inminente necesidad de realizar una propuesta educativa de convergencia, a la luz de los cambios realizados en años recientes a las políticas de educación superior, a fin de identificar cómo se constituye la educación a distancia en Brasil, y qué posibilidades existen para una educación de convergencia entre estas dos modalidades.

Para finalizar este monográfico, Moisés Ramírez et al, analizan desde un enfoque mixto, el uso que hacen de las TIC los profesores universitarios. Parten de la importancia de las TIC en la docencia universitaria, y nos comparten las percepciones y valoraciones que realizan los docentes con respecto al uso y dominio de las TIC en el ámbito educativo. Asimismo, nos muestran la opinión que tienen los docentes con relación a la labor docente con el empleo de las TIC, su incorporación en el aula y al proceso educativo con TIC. Resaltando su transcendencia de estas, en actividades académicas -investigación, vinculación, gestión, docencia, ejecución y tutoría.

\section{Guadalupe Aurora Maldonado Berea} Universidad Veracruzana 